\title{
COMPENSAÇÃO AMBIENTAL APLICADA NA ARBORIZAÇÃO URBANA DA CIDADE DE VITÓRIA - ES.
}

Flávio do Nascimento Coelho ${ }^{1}$, Maria Alice Soares Linhares ${ }^{2}$

\section{RESUMO}

O presente trabalho trata da compensação ambiental aplicada na arborização urbana da cidade de Vitória, para casos em que a construção e/ou reforma de imóveis, impliquem em remoção ou relocação de exemplares em bom estado vegetativo e fitossanitário. O valor da compensação ambiental foi calculado com base nos custos de produção de muda, plantio, poda anual, tratamento fitossanitário anual e retirada, acrescido de uma taxa de até $50 \%$ do valor total, que levou em consideração o valor ambiental de cada exemplar conforme a sua espécie, porte, localização, estado vegetativo e fitossanitário. Foi aplicada a compensação ambiental em 12 casos, nos quais a retirada de vegetais foi inevitável, o que resultou em 25 exemplares arbóreos retirados. As principais espécies retiradas foram Albizia lebbeck Albizia (32\%); Licania tomentosa - Oiti (24\%) e Caesalpinia peltophoroides - Sibipiruna (16\%). O principal motivo para retirada foi abertura de garagem. O termo de compensação ambiental demonstrou ser uma ferramenta eficiente para o gerenciamento de árvores urbanas, uma vez que destaca a importância de se incluir o componente arbóreo existente na cidade nos novos projetos imobiliários a serem implementados.

Palavras-chave: Arborização urbana, Compensação ambiental, Avaliação de árvores urbanas.

\footnotetext{
${ }^{1}$ Biólogo, Secretaria Municipal de Meio Ambiente - PMV, Vitória, Espírito Santo, coelhofn@hotmail.com

2 Bióloga, Secretaria Municipal de Meio Ambiente - PMV, Vitória, Espírito Santo, mariaalicelinhares@yahoo.com.br
} 


\section{SUMMARY}

The present work expounds the environment compensation to the urban arborization of the city of Vitória - Espírito Santo - Brazil, for cases where the construction and/or reform of property, imply in removal or relocation of trees in vegetative and health good conditions. The environment compensation value was calculated according to seedling production costs, plantation, annual pruning, pests and diseases annual treatment and tree remotion, increased of a tax of up to $50 \%$ of the total value, considering the environment value of each tree unit, according its species, size, place, vegetative and helth condition. The environment compensation was applied in 12 cases, in which the tree remotion was inevitable, resulting in 25 tree remotions. The main removed species were Albizia lebbeck; Caesalpinia peltophoroides and Licania tomentosa. The main reason for the remotion was cases for garage constructions. The environment compensation term has demonstrated to be an efficient tool for the management of urban trees, pointing out the importance of including the tree component in the implementation of new construction projects in the city .

Key-words: Urban arborization, Environment compensation, Urban trees evaluation 


\section{INTRODUÇÃO}

Ao contrário dos ambientes naturais, as cidades apresentam artificialidades, como forte impermeabilização do solo, abundância de materiais altamente refletores, absorventes e transmissores de energia, excessivo consumo de energia e matéria, com correspondente geração de resíduos, poluição atmosférica, hídrica, sonora e visual. Tais características afetam negativamente o ambiente urbano, e em conseqüência a qualidade de vida das pessoas (MILANO, 2000). No Brasil, os centros urbanos constituem locais de grande desconforto térmico, que é agravado pelo clima tropical com forte nível de insolação durante todo ano. Neste contexto, torna-se fundamental a manutenção e/ou implantação de áreas verdes urbanas, para diminuir estes extremos de calor. Além disso, a arborização também é responsável por trazer benefícios em relação à saúde e bem estar da população, diminuição da poluição e a valorização dos imóveis. (GOLD, 1977; GREY \& DENEKE, 1978; BARTENSTEIN, 1981; DETZEL, 1993).

Vitória, a capital do estado do Espírito Santo, apresenta já urbanizada a maior parte do seu território, que é constituído de uma parte continental e outra insular. O processo de urbanização local ao longo do tempo, caracterizou-se pela superposição de tecidos urbanos de diferentes épocas e pela conquista de terra para expansão urbana sobre os baixios, manguezais e o próprio mar. Características do pouco espaço disponível, a cidade apresenta ruas e passeios estreitos, falta de espaços para estacionamento de veículos, inexistência de afastamento predial, fatores relacionados a falta ou deficiência de planejamento urbano que, aliados à baixa qualidade dos solos locais, sempre dificultaram ou impediram o desenvolvimento da necessária arborização na cidade (PDA / PMV, 1992).

Além desses fatores, a competição natural da arborização pública com mobiliários urbanos como postes, placas de trânsito, fiação elétrica e telefônica, bancas de jornal, encanamentos, estacionamento, entradas de garagens e outros, foi agravada na última década por um verdadeiro "boom" de construções de condomínios residenciais e comerciais em alguns bairros nobres. Na maioria dos casos, os novos projetos não levam em consideração o componente arbóreo existente, o que acarreta um grande número de solicitações de retirada de árvores em bom estado vegetativo e fitossanitário, sendo, em muitos casos, exemplares de grande porte e bem adaptados ao ambiente. Diante desta realidade, foi desenvolvido o presente trabalho que visa aplicar termos de compensação ambiental para casos em que a construção e/ou reforma de imóveis, impliquem em remoção ou relocação de exemplares arbóreos, buscando assim reduzir as solicitações de retirada de 
árvores para implementação de novos empreendimentos imobiliários, levar os empreendedores e profissionais da construção civil a valorizar os componentes arbóreos da cidade e divulgar, por meio da educação ambiental, os benefícios da arborização urbana.

\section{MATERIAIS E METÓDOS}

\section{Base legal}

A base legal do trabalho foi amparada no Decreto $n^{\circ} 11.975$ de 2004, que regulamenta a Lei no 6080 de 2003 que trata do Código de Posturas e Atividades Urbanas. Neste documento foi incluído o Artigo 154 que trata da compensação ambiental de elementos da arborização urbana, caso a construção e/ou reforma de imóveis impliquem em remoção ou relocação da mesma.

\section{Valor e pagamento da compensação ambiental}

O valor da compensação foi calculado em reais, com base nos custos operacionais de cada exemplar arbóreo (CO) que levou em consideração: Produção de muda (PM), Plantio (P), Poda anual (PA), Tratamento fitossanitário anual (TF) e Retirada (R), sendo que PA e TF são multiplicados pela idade estimada do vegetal (i), acrescido de uma taxa de até $50 \%$ do valor total, que levou em consideração o Valor Ambiental (VA) de cada exemplar conforme a sua espécie, porte, localização, estado vegetativo e fitossanitário, conforme equação (1) abaixo:

$$
C O(P M+P+P A x i+T F x i+R)+V A
$$

A idade do vegetal foi estimada pelos registros de data de plantio quando disponíveis e pelo diâmetro do tronco do vegetal na altura do peito.

O pagamento da compensação ambiental foi feito por meio da conversão do valor em reais para mudas e/ou grama, com padrões e espécies determinadas pelos técnicos da secretaria do meio ambiente, e doadas ao horto municipal. Após o pagamento da compensação ambiental, as retiradas eram executadas (Anexos 1 e 2).

\section{Educação ambiental}

No processo de aplicação do termo de compensação, foi discutida com os engenheiros, arquitetos e empreendedores, a real necessidade de retirada do vegetal, sua 
possível adequação ao projeto arquitetônico e a necessidade de se preservar o patrimônio arbóreo da cidade. Na ocasião foi distribuída a cartilha de arborização da cidade de Vitória, que disponibiliza orientações a respeito dos benefícios da arborização, cuidados com as mudas e árvores em geral, bem como os serviços oferecidos pela secretária do meio ambiente, por meio do sistema Fone Verde.

\section{RESULTADOS E DISCUSSÃO}

Durante o período de dezembro de 2005 a setembro de 2006, foram feitas através do sistema Fone Verde 869 solicitações de vistorias de árvores, sendo realizados vários serviços como poda, abertura de área livre, retiradas e substituições de árvores. Em 12 casos, nos quais não havia indicativos técnicos que justificassem a retirada do vegetal, foi aplicado o termo de compensação ambiental, que resultou na retirada de 25 exemplares arbóreos. As principais espécies retiradas foram Albizia lebbeck - Albizia (32\%); Licania tomentosa - Oiti (24\%) e Caesalpinia peltophoroides - Sibipiruna (16\%) (Figura 01).

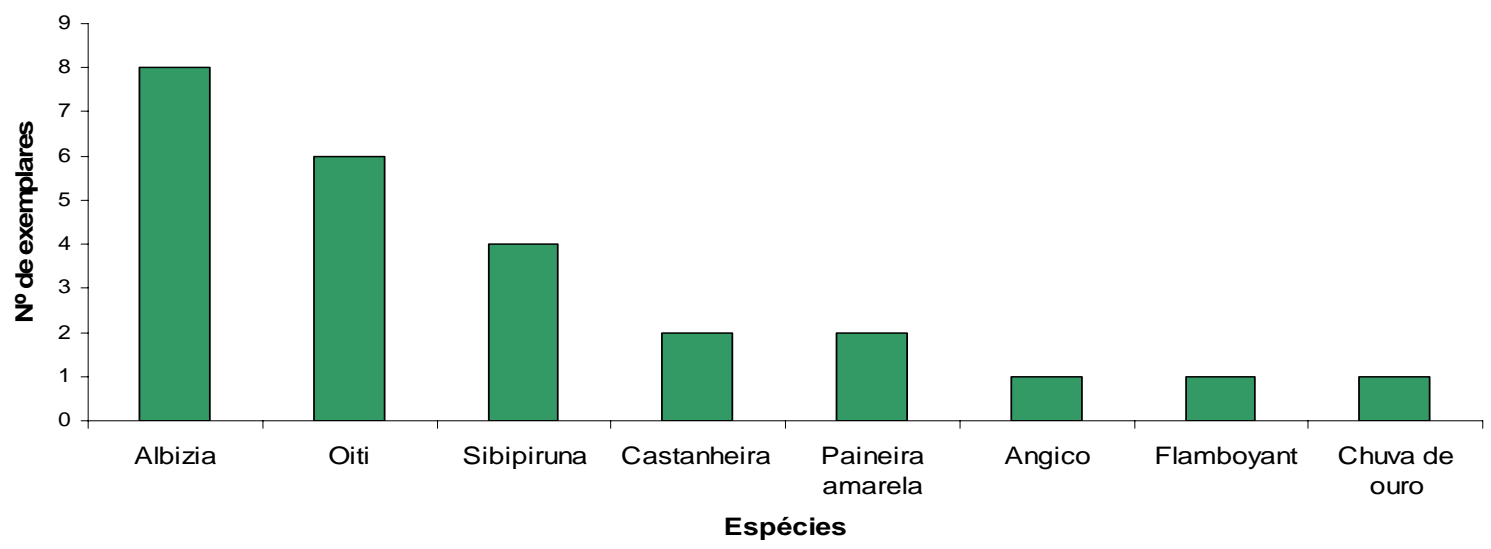

Figura 01 - Principais espécies arbóreas retiradas na aplicação do termo de compensação ambiental da cidade de Vitória.

Os principais motivos para retirada de árvores foram incompatibilidade com o novo projeto em que foram previstas novas aberturas de garagem (Figura 02). 


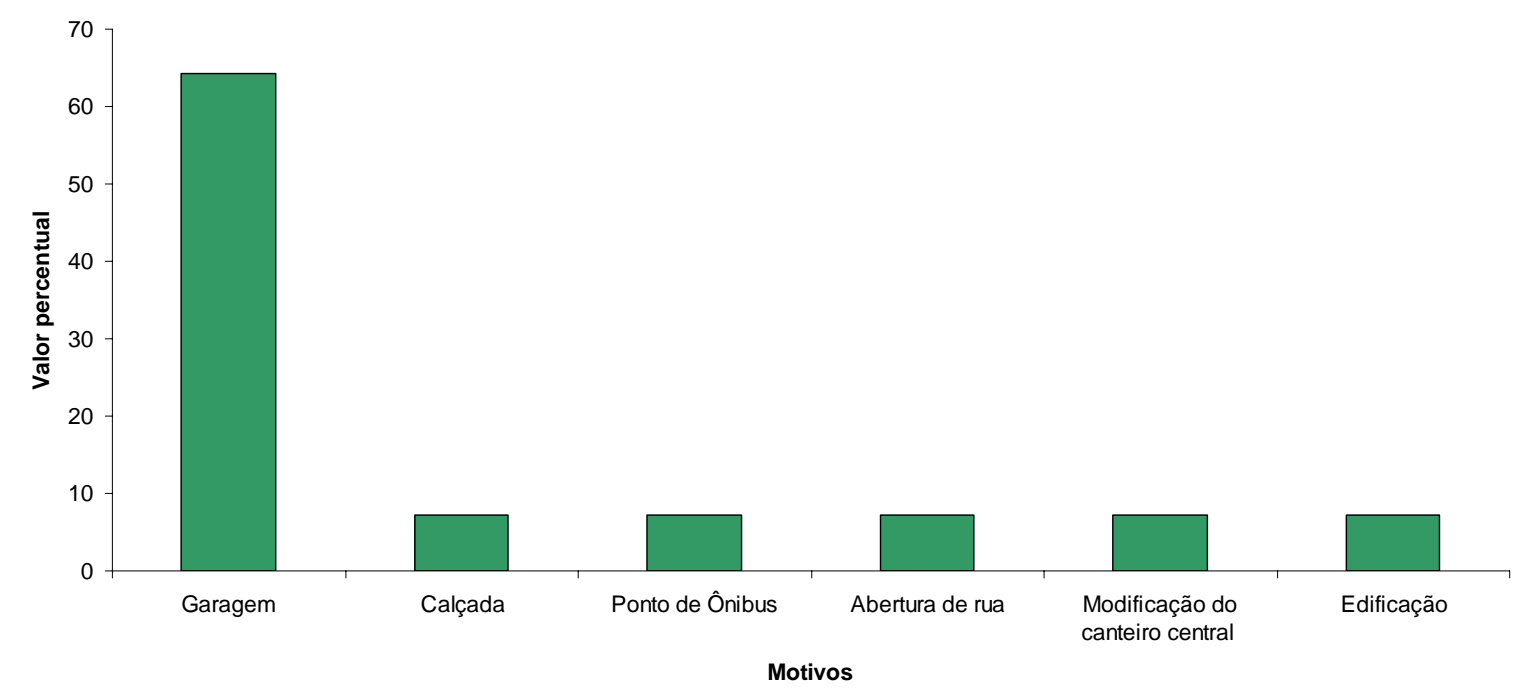

Figura 02 - Principais motivos para retiradas de árvores na aplicação do termo de compensação ambiental da cidade de Vitória.

O valor do pagamento da compensação ambiental resultou no recebimento de 6.562 mudas de árvores e / ou espécies ornamentais e $2.835 \mathrm{~m}^{2}$ de grama (Tabela 01 ). 
Tabela 01 - Total de mudas de árvores, espécies ornamentais e grama recebidos na aplicação do termo de compensação ambiental da cidade de Vitória.

\begin{tabular}{|l|l|l|l|}
\hline Data & Quantidade & $\mathrm{m}^{2} /$ unid. & Espécie recebida \\
\hline $29 / 12 / 05$ & 450 & $\mathrm{~m}^{2}$ & Zoyzia japonica speud \\
\hline $29 / 11 / 05$ & 450 & $\mathrm{~m}^{2}$ & Zoyzia japonica speud \\
\hline $10 / 3 / 06$ & 40 & unid. & T. roseo alba, L. speciosa, C. viminalis, D.decary \\
\hline $26 / 3 / 06$ & 60 & unid. & T. roseo alba, T. granulosa \\
\hline $26 / 3 / 06$ & 295 & $\mathrm{~m}^{2}$ & Zoyzia japonica speud \\
\hline $10 / 3 / 06$ & 24 & unid. & Alcantarea imperialis \\
\hline $14 / 6 / 06$ & 1640 & $\mathrm{~m}^{2}$ & Zoyzia japonica speud \\
\hline $1 / 8 / 06$ & 3270 & unid. & Gloxinia sy/vatica \\
\hline $15 / 8 / 06$ & 55 & unid. & Tabebuia pentaphylla \\
\hline $5 / 9 / 06$ & 5 & unid. & Bismarckia nobilis \\
\hline $30 / 8 / 06$ & 2908 & unid. & Crossandra infundibuliformes \\
\hline $25 / 8 / 06$ & 110 & unid. & C. viminalis, L. speciosa, T. Roseo alba, C. fistula \\
\hline 05/09/06 & 90 & unid. & Callistemom viminalis \\
\hline Total de mudas 6.562 & Unidades; Total de grama 2.835 m $^{2}$ \\
\hline
\end{tabular}

O trabalho de educação ambiental desenvolvido no processo de aplicação do termo de compensação atingiu diretamente, 30 profissionais da área de construção civil. O que resultou em maior sensibilização desses atores urbanos para a necessidade da manutenção das áreas verdes do município.

O processo de aprovação de novos projetos no município também foi modificado, pois, a partir deste trabalho, foi elaborada uma instrução normativa, que exige que todos os novos projetos sejam vistoriados com o objetivo de verificar se há incompatibilidade com os exemplares arbóreos existentes.

\section{RESULTADOS E DISCUSSÃO}

$\mathrm{Na}$ aplicação da compensação ambiental, não foi observada nenhuma correlação com a espécie, porte ou estado vegetativo e fitossanitário do vegetal, o que demonstra que os motivos para solicitação de retirada estão relacionados à concepção do projeto arquitetônico desenvolvido e não diretamente a inadequações da arborização urbana. 
O cálculo do valor monetário por exemplar utilizado foi considerado satisfatório, no sentido que cobriu os custos operacionais municipais, no entanto, quantificar o valor ambiental de uma árvore urbana é uma tarefa muito difícil e quase sempre subjetiva. Segundo DETZEL, 1992 o fato de muitas pessoas pensarem que os benefícios propiciados pela árvore são tantos que a tornam insubstituível, atribuindo-lhe um valor muito alto, torna-a intangível em termos monetários. A situação inversa também pode ocorrer. Dependendo do nível de informação, certas pessoas podem desmerecer tanto a existência de uma árvore a ponto de lhe causar danos ou mesmo suprimi-la.

Iniciativas de avaliação monetária de árvores individuais datam do século passado. Os métodos aplicados, a principio arbitrários, foram aos poucos incorporando critérios técnicos como estimativas de idade, espécie, porte, localização e condições fitossanitárias do vegetal (GREY \& DENEKE, 1978; KIELBASO, 1979).

Segundo a International Society of Arboriculture (ISA, 2006), apesar da responsabilidade de corrigir conflitos causados pela arborização não poder ser atribuída a particulares, nos casos em que o conflito resultar de ações do proprietário que ferem diretrizes municipais estabelecidas, cabe ao proprietário arcar com os custos da ação corretiva. A avaliação monetária das árvores de uma cidade justifica-se pelo fato de fornecer dados importantes ao planejamento, implantação, manutenção e remoção da arborização, por estabelecer valores de multas por danos causados às árvores e estabelecer parâmetros de indenizações em questões legais ou jurídicas (DETZEL, 1992 ).

O trabalho de educação ambiental proporcionou maior integração entre profissionais da construção civil e a coordenação de arborização e paisagismo da secretaria de meio ambiente. Tal ferramenta é de fundamental importância para o gerenciamento de árvores urbanas e o planejamento da cidade, o que está em conformidade com MILANO, 2000 que cita as campanhas de conscientização pública como um instrumento imprescindível para o sucesso de programas ambientais desenvolvidas pelo poder público.

\section{CONCLUSÕES}

O termo de compensação ambiental demonstrou ser uma ferramenta eficiente para o gerenciamento de árvores urbanas, uma vez que destaca a importância de se incluir o componente arbóreo existente na cidade nos novos projetos imobiliários a serem implementados. Sua aplicação permitiu a discussão dos técnicos da Secretária de Meio Ambiente com os projetistas, executores e empreendedores, resultando em maior 
sensibilização desses atores urbanos para a necessidade da manutenção das áreas verdes do município.

\section{REFERÊNCIAS BIBLIOGRÁFICAS}

BARTENSTEIN, F. The future os urban forestry. J. Arb. , 7(10) : $261-7,1981$.

DALCIN, E. \& MILANO, M. S. Arborização de vias públicas. Rio de Janeiro, 2000.

DETZEL, V.A. Avaliação Monetária de Árvores Urbanas. In: Curso sobre Arborização Urbana. Paraná, 1992. p120-132.

GOLD, S. M. Social and economics benefits of trees in cities. J. For., 75(2):84 - 87,1977.

GREY, J. J. \& DENEKE, F. J. Urban forestry. New York, Jonh Wiley, 1978. 279pp.

ISA, 2006. International Society of Arboriculture . Disponível em <http.//www.isa-arbor.com>

KIELBASO, J. J. HASTON, G. \& PAWL, D. Municipal tree management in the U. S. 1980. J. Arb., 8(10):253 - 257, 1982.

MILANO, M. S. Avaliação quali-quantitativa e manejo de arborização urbana: exemplo de Maringá - Pr. Curitiba, UFPr,1988. 120p.

PREFEITURA MUNICIPAL DE PORTO ALEGRE. Plano diretor de arborização de vias públicas. Porto Alegre, 2000.

PREFEITURA MUNICIPAL DE VITÓRIA. Plano diretor de arborização e áreas verdes. Vitória, 1992. 97p. 


\section{ANEXOS}

Anexo 1 - Exemplo do termo de compensação aplicado no município de Vitória.

\begin{tabular}{|c|c|c|}
\hline क्जा & $\begin{array}{l}\text { Prefeitura Municipal de Vitória } \\
\text { Secretaria Municipal de Meio Ambiente }\end{array}$ & $\begin{array}{l}\text { TERMO DE COMPENSAÇÃO } \\
\text { AMBIENTAL } \\
\text { No XXX/2006 }^{\circ}\end{array}$ \\
\hline \multicolumn{3}{|c|}{$\begin{array}{l}\text { Através deste instrumento, a Empres } \\
\text { retirada de xx exemplares arbóreo do } \\
\text { consta do documento nos termos da } \\
\text { art. } 154 \text {, e de acordo com a proposta } \\
\text { Obs.: Fone Verde } \mathbf{n}^{0} \text { xxxxxxx/2006 }\end{array}$} \\
\hline \multicolumn{3}{|c|}{$\begin{array}{l}\text { Proposta Técnica: } \\
\text { Doação de mudas das espécies relacionadas abaixo para o exemplar arbóreo retirado - } \\
\text { xxxxxxx, que serão destinadas a ações de melhoria da arborização e paisagismo urbano } \\
\text { em Vitória e em atenção às seguintes indicações quanto à(s) espécie(s) e padrões } \\
\text { técnicos: }\end{array}$} \\
\hline $\begin{array}{r}\text { Quant } \\
\text { xx }\end{array}$ & $\begin{array}{ll}\text { aproximada } & \text { Nome popu } \\
x x x x x x x x x x & x x x x x x x x x x x\end{array}$ & $\begin{array}{l}\text { Nome científico } \\
x x x x x x x x x x x\end{array}$ \\
\hline \multicolumn{3}{|c|}{$\begin{array}{l}\text { Obs.: A quantidade de mudas estabelecidas, eqüivale ao valor de } \mathbf{R} \mathbf{\$} \mathbf{x x x} \mathbf{x x} \text {, referente } \\
\text { ao custo operacional que a SEMMAM tem relativo à produção, plantio, poda/ano, } \\
\text { tratamento fitossanitário/ano e retirada, acrescido do valor ambiental, que varia até o } \\
\text { máximo de } 50 \% \text {, dependendo da espécie e do estado vegetativo do vegetal. } \\
\text { As mudas deverão ser adquiridas conforme padrões estabelecidos pela Secretaria } \\
\text { Municipal de Meio Ambiente, a saber: } \\
\text { om estado vegetativo e fitossanitário } \\
\text { lura das mudas: } \mathbf{1 , 8 0 m}\end{array}$} \\
\hline \multicolumn{3}{|c|}{ OBS.: FAVOR ANEXAR CÓPIA DA NOTA FISCAL. } \\
\hline \multicolumn{3}{|c|}{ Vitória, 25 de agosto de 2006.} \\
\hline
\end{tabular}


Anexo 2 - Exemplo de recibo do termo de compensação aplicado no município de Vitória.

\begin{tabular}{|c|c|c|}
\hline inting & $\begin{array}{l}\text { Prefeitura Municipal de Vitória } \\
\text { Secretaria Municipal de Meio Ambiente }\end{array}$ & $\begin{array}{l}\text { TERMO DE COMPENSAÇÃO } \\
\text { AMBIENTAL } \\
N^{\circ} \text { XXX/2006 }\end{array}$ \\
\hline \multicolumn{3}{|c|}{$\begin{array}{l}\text { Recebemos da Empresa XXXX, os vegetais abaixo discriminados, referente a } \\
\text { compensação ambiental - Termo de Compensação } \text { n }^{\circ} \text { XXX/2006, (conforme consta do } \\
\text { documento nos termos da Lei Municipal no 6080/2003 e decreto 1197/2004, art. 154), pela } \\
\text { retirada de } 01 \text { (um) exemplar arbóreo de xxxxxxxx da arborização pública, conforme } \\
\text { consta do documento nos termos da Lei Municipal no 6080/2003 e Decreto 11.975/2004, } \\
\text { art. } 154 \text { e de acordo com a proposta técnica abaixo: }\end{array}$} \\
\hline \multicolumn{3}{|c|}{ Obs.: Fone Verde $n^{\circ}$ xxxxxxx/2006 } \\
\hline Quan & Nome vulgar & Nome científico \\
\hline$x x x x x x$ & $x x x x x x x x x x x$ & $x x x x x x x x x x x x x x$ \\
\hline \multicolumn{3}{|c|}{$\begin{array}{l}\text { As mudas estão de acordo com os padrões estabelecidos pela Secretaria Municipal de } \\
\text { Meio Ambiente: }\end{array}$} \\
\hline$\bullet$ & $\begin{array}{l}\text { Bom estado vegetativo e fitossanitário. } \\
\text { Altura da muda: } 1,80 \mathrm{~m}\end{array}$ & \\
\hline \multicolumn{3}{|c|}{ OBS.: FAVOR ANEXAR CÓPIA DA NOTA FISCAL, no valor de xxxxxxx. } \\
\hline \multicolumn{3}{|c|}{ Vitória, xx de xxxxxxx de 2006.} \\
\hline $\operatorname{Res}$ & sponsável pelo Horto Municipal & \\
\hline
\end{tabular}

